\title{
TWO-WAY COUPLED ELECTRO-THERMAL ANALYSIS OF FGM SYSTEM CALCULATED BY THE NEW LINK FINITE ELEMENT
}

\section{Juraj Paulech ${ }^{1}$, Vladimír Kutišs ${ }^{1}$, Juraj Hrabovský ${ }^{1}$, Justín Murín ${ }^{1}$, Roman Gogola ${ }^{1}$, Tibor Sedlár ${ }^{1}$, Gabriel Gálik ${ }^{1}$}

\footnotetext{
${ }^{1}$ Department of Applied Mechanics and Mechatronics, Institute of Automotive Mechatronics, Faculty of Electrical Engineering and Information Technology, Slovak University of Technology in Bratislava Ilkovičova 3, 81219 Bratislava, Slovakia e-mail: juraj.paulech@stuba.sk
}

Keywords: Thermoelectric Analysis, FGM Link Finite Element, ANSYS.

\begin{abstract}
The paper deals with derivation process of new FEM equations for steady thermoelectric two-way coupled analysis of link conductor made of Functionally Graded Material (FGM). One example of coupled analysis will be introduced to demonstrate accuracy and effectiveness of our new approach in computer modelling of such systems.
\end{abstract}




\section{INTRODUCTION}

Nowadays, new materials are necessary for sophisticated structures like MEMS systems, advanced electronic devices, etc. Computer modelling of such complex systems, like structures with spatial variation of material properties (e.g. FGM) are, using commercial FEM code with classic elements, needs remarkable effort during preparation phase and sufficient computer equipment for solution phase because of necessity the numbers of elements and material models.

Finite elements for electric-thermal analyses of FGM materials considering Joule heat have been developed in [1]. This paper deals with derivation of new link finite element for twoway coupled static thermoelectric analyses considering Joule heat and also thermoelectric effects like Seebeck and Peltier effects. These effects describe direct conversion of thermal energy into electric energy (Seebeck effect) and conversion of electric energy into the temperature difference within the system (Peltier effect).

Let us consider straight link conductor, the conductor is a slender construction. Let the conductor is made of a mixture of two or more materials so its thermal, electric and thermoelectric material properties change according to chosen function. Under these conditions, we can consider one-dimensional system of differential equations and original method further explained in [2] for solving the differential equations for thermoelectric coupled analysis.

\section{HOMOGENIZATION OF MATERIAL PROPERTIES}

Let us consider FGM conductor with length $L[\mathrm{~m}]$, and rectangular cross-section area $A\left[\mathrm{~m}^{2}\right]$ (height $h[\mathrm{~m}]$ and width $b[\mathrm{~m}]$ ) with nodes symbolically denoted " 0 " and "L", see Figure 1.

Let the conductor is made of mixture of two component materials - matrix (index $m$ ) and fibre (index $f$ ). Let the change of material properties is only in longitudinal and transversal direction (2D change). Let the thermal conductivities $\lambda_{m}$ and $\lambda_{f}$, and electric conductivities $\sigma_{m}$ and $\sigma_{f}$ are known values for matrix and fibre, respectively. Then, according to the homogenization process described in [1] we can calculate homogenized thermal and electric conductivities for whole conductor. These homogenized material properties need to be polynomials.
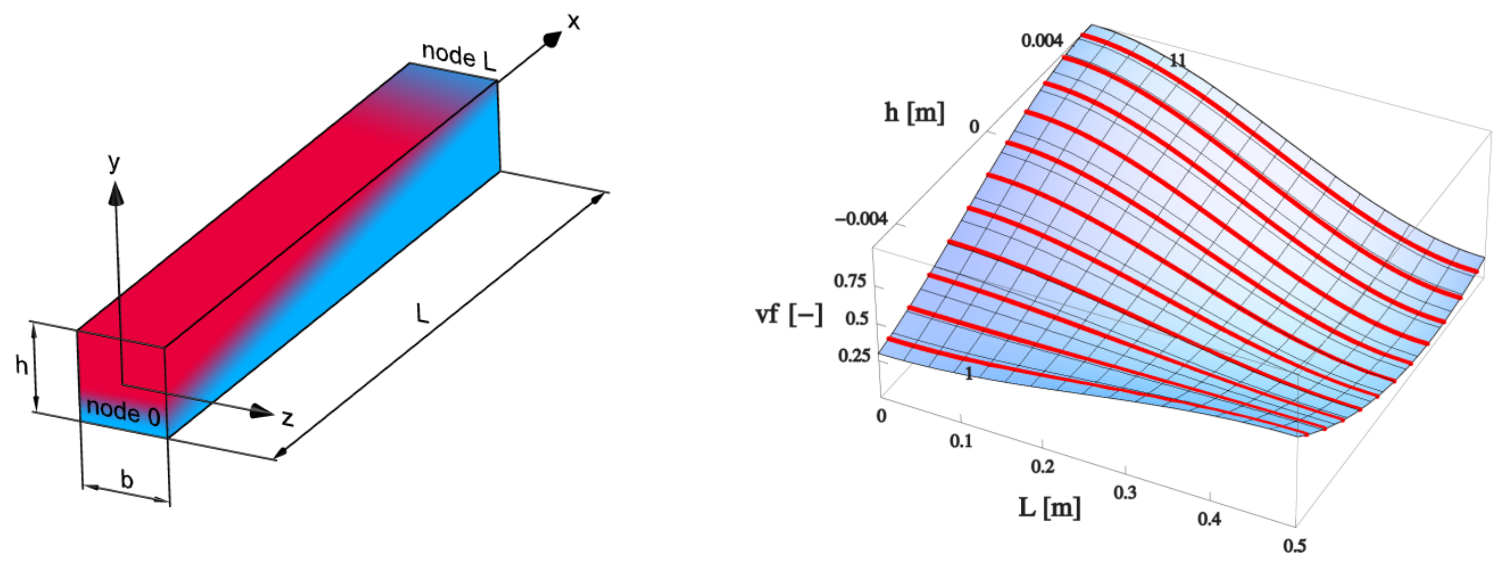

Figure 1: FGM conductor (left) and fibre volume fraction in the conductor - division into layers (right) 
Homogenization process supposes that volume fractions of matrix and fibre are known and have also polynomial form, see Figure 1. Then, homogenization includes two steps:

- In the first step the real link is transformed to a multilayered link, real link is divided into chosen number of layers in transversal direction $(N=11$ for the case shown in Figure 1). The extended mixture rule [3] is used for calculation of material properties of the layers. Then, each layer will have constant volume fraction and material properties of the constituents through its height, see Figure 2. Polynomial variation of these parameters will appear only in the longitudinal direction of the layers.

- In the next step, these layers will be used for calculation of homogenized material properties for whole link (see Figure 2) according to laminate theory described in [4]. This homogenized material properties will interpret material changes in the whole FGM link and they will have polynomial form with just one independent variable (x).

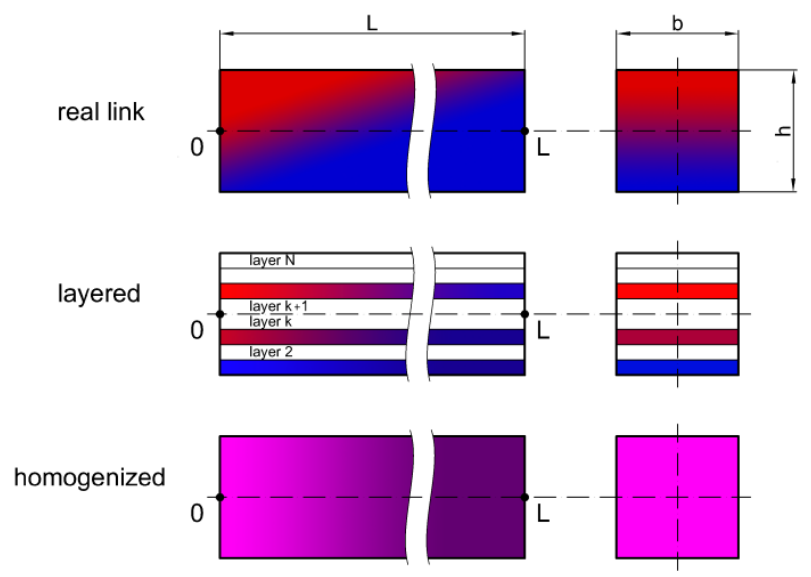

Figure 2: Graphic form of the homogenization process

For thermoelectric coupling that results in Seebeck and Peltier effects, Seebeck coefficient $\alpha(x, y)$ of the whole conductor needs to be known. This material property cannot be calculated according to the homogenization process based on extended mixture rule and laminate theory, because this material property is not given according to volume fractions of individual material components (expect the Joule heat, thermoelectric effects are significant especially for semiconductor materials and the behaviour of the semiconductors is given according to atomic structure, not according to volume fraction of individual admixtures). Moreover, the value of Seebeck coefficient can also be negative number. The determination of final Seebeck coefficient according to material properties of individual components is beyond the scope of this article. For our model case the final Seebeck coefficient will be chosen as a polynomial function $\alpha(x)$ for longitudinal direction of the homogenized conductor.

\section{MATHEMATICAL BACKGROUND FOR TWO-WAY COUPLED THERMOELECTRIC ANALYSIS}

Thermoelectric and electric-thermal effects like Joule heat, Seebeck, Peltier and Thomson effects are described by set of two thermoelectric constitutive equations (static analysis) [5]:

$$
\begin{gathered}
\mathbf{q}=\llbracket \Pi \rrbracket \cdot \mathbf{J}-\llbracket \lambda \rrbracket \cdot \nabla T \\
\mathbf{J}=\llbracket \sigma \rrbracket \cdot(\mathbf{E}-\llbracket \alpha \rrbracket \cdot \nabla T)
\end{gathered}
$$

where $\mathbf{q}\left[\mathrm{Wm}^{-2}\right]$ is heat flux vector, $\mathbf{J}\left[\mathrm{Am}^{-2}\right]$ is electric current density vector, $\llbracket \Pi \rrbracket[\mathrm{V}]$ is Peltier coefficient matrix, $\llbracket \lambda \rrbracket\left[\mathrm{Wm}^{-1} \mathrm{~K}^{-1}\right]$ is thermal conductivity matrix, $T[\mathrm{~K}]$ is absolute 
temperature, $\mathbf{E}\left[\mathrm{Vm}^{-1}\right]$ is electric field intensity vector, $\llbracket \sigma \rrbracket\left[\mathrm{Sm}^{-1}\right]$ is electric conductivity matrix and $\llbracket \alpha \rrbracket\left[\mathrm{VK}^{-1}\right]$ is Seebeck coefficient matrix.

These constitutive equations are coupled by set of governing equations for static thermal and electric fields:

$$
\begin{aligned}
& \nabla \cdot \mathbf{q}=P \\
& \nabla \cdot \mathbf{J}=0
\end{aligned}
$$

where $P\left[\mathrm{Wm}^{-3}\right]$ is heat generation per volume unit.

In general, we can write for electric field intensity, Peltier coefficient and heat generation:

$$
\begin{array}{cc}
\mathbf{E}=-\nabla \varphi & P=P_{J}+P_{\text {aux }} \\
\llbracket \Pi \rrbracket=T \llbracket \alpha \rrbracket & P_{J}=\llbracket \sigma \rrbracket^{-1} \mathbf{J}^{2}
\end{array}
$$

where $\varphi[\mathrm{V}]$ is electric potential, $P_{J}\left[\mathrm{Wm}^{-3}\right]$ is Joule heat per volume unit and $P_{a u x}\left[\mathrm{Wm}^{-3}\right]$ is auxiliary heat generation per volume unit.

Applying (1) and (3) into (2) we can write for 1D system (longitudinal direction $x$ ):

$$
\begin{gathered}
\frac{d}{d x}[T(x) \alpha(x) J(x)]-\frac{d}{d x}\left[\lambda(x) \frac{d T(x)}{d x}\right]=\frac{J^{2}(x)}{\sigma(x)}+P_{a u x}(x) \\
\frac{d}{d x}\left[\sigma(x) \frac{d \varphi(x)}{d x}\right]+\frac{d}{d x}\left[\sigma(x) \alpha(x) \frac{d T(x)}{d x}\right]=0
\end{gathered}
$$

\section{DERIVATION OF NEW FGM EQUATIONS FOR FGM LINK CONDUCTOR}

We can use the method for solving 1D differential equation with non-constant coefficients and with right-hand side described in [2] for our system of equations (4). But all non-constant coefficients on the left-hand side and right-hand side itself have to be in polynomial form (it is the condition of used approach). So general formulation of one-dimensional differential equation suitable for the method has the form:

where:

$$
\sum_{u=0}^{m} \eta_{u}(x) y^{(u)}(x)=\sum_{j=0}^{g} \varepsilon_{j} a_{j}(x)
$$

$m \quad-$ degree of the differential equation

$y(x) \quad-$ unknown function of independent variable $x$

$y^{(u)}(x) \quad-u^{\text {th }}$ derivation of the unknown function

$\eta_{u}(x) \quad-$ polynomial variable coefficient for $u^{\text {th }}$ derivation on the left-hand side of the differential equation

$g-$ degree of a polynomial on the right-hand side of the differential equation

$\varepsilon_{j} \quad-$ constant coefficient for $j^{\text {th }}$ power of the right-hand side polynomial

$a_{j}(x)=\frac{x^{j}}{j !} \quad$ - auxiliary function for the right-hand side polynomial formulation

at which $x \in\langle 0 ; L\rangle$, where $L$ is the length of considered interval of unknown solution.

Considering function $a_{j}(x)$ for formulation the polynomials, we can write general rules for derivation and integration of such function:

$$
\begin{gathered}
a_{j}^{\prime}(x)=a_{j-1}(x) \\
\int_{0}^{x} a_{j}(x) d x=a_{j+1}(x)
\end{gathered}
$$


According to [2] the solution of the differential equation (5) has the form:

where:

$$
y(x)=\sum_{i=0}^{m-1} y_{0}^{(i)} c_{i}(x)+\sum_{j=0}^{g} \varepsilon_{j} b_{j+m}(x)
$$

$y_{0}^{(i)} \quad i^{\text {th }}$ derivation of the function $y$ in $x=0$, thus $y_{0}^{(i)}=\left.y^{(i)}(x)\right|_{x=0}$

$c_{i}(x) \quad$ function for uniform solution of the differential equation

$b_{j+m}(x) \quad$ function for particular solution of the differential equation

And derivation of the solution (7) is in the form:

$$
y^{(u)}(x)=\sum_{i=0}^{m-1} y_{0}^{(i)} c_{i}{ }^{(u)}(x)+\sum_{j=0}^{g} \varepsilon_{j} b_{j+m}^{(u)}(x) \quad u=\{0, m-1\}
$$

The solution of differential equation (7) lies in determining the functions generally labelled $c(x)^{1}$ and $b(x)$ that appear in the solution.

The result of calculation of the differential equation with variable coefficients and the right-hand side is the solution according to equation (7). It should be noted that this is a solution for selected point $x$ of the considered interval of independent variable, so the program is designed for calculation of values $c_{i}(x)$ in given point $x$ for $i=\{0, m-1\}$ where $m$ is the degree of the differential equation. Then the values $b_{j+m}(x)$ for selected point $x$ are also calculated for $j=\{0, g\}$ where $g$ is degree of the right-hand side polynomial of the differential equation. It means that functions $c(x)$ and $b(x)$ cannot be calculated analytically but only at discrete points $x$, where $x$ is from interval $\langle 0 ; L\rangle$.

The program algorithm, also described in [2], efficiently calculates matrixes of discrete values $c_{i}^{(u)}(x)$ and $b_{j+m}^{(u)}(x)$ for user defined point $x$ where $u=\{0, m-1\}$ represents derivation for calculations according to (8).

It should be noted that the polynomial function of the right-hand side of the differential equation itself does not enter into the calculation. For the calculation of $c(x)$ and $b(x)$ it is only necessary to know the degree of the right-hand side polynomial. The values of the coefficients $\varepsilon_{j}$ of the right-hand side only enter into the final equation - solution (7) or derivation (8), respectively.

Programming code offers the possibility of automatic uniform interval division calculation, and the values of $c(x)$ and $b(x)$ for interval dividing points are included in the output. So during single run of the program we can get all the necessary values to solve the differential equation also for selected internal points in the interval $x \in(0 ; L)$, in our case for conductor inner region.

This program for solving differential equations is used in process of deriving new FEM equations for two-way coupled thermoelectric analysis in FGM link conductor mentioned above. Figure 3 shows geometry and physical quantities used during the derivation process.

\footnotetext{
${ }^{1}$ denoting is symbolical - general; correctly it should be mentioned that it is a set of values $c_{i}(x)$ within the range $i=\{0, m-1\}$; it is also similar for $b_{j}(x)$ with the range $j=\{0, m-1\}$
} 


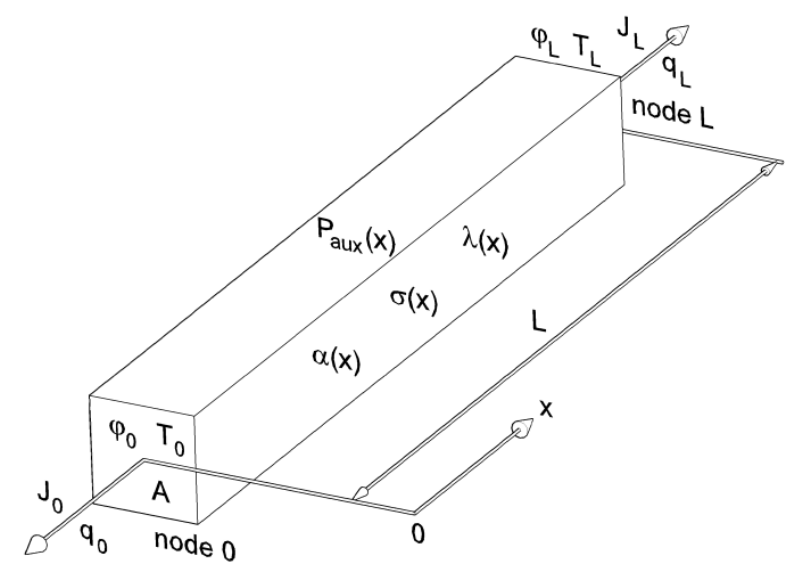

Figure 3: Two-nodal conductor for thermoelectric analysis

Let us write the governing function (4) for heat flux in the form suitable for calculation according [2]. Comparing the general form of the differential equation (5) with governing function (4) in expanded form:

$$
\begin{gathered}
\sum_{u=0}^{m} \eta_{u}(x) y^{(u)}(x)=\sum_{j=0}^{g} \varepsilon_{j} a_{j}(x) \\
-\lambda(x) T^{\prime \prime}(x)+\left[\alpha(x) J(x)-\lambda^{\prime}(x)\right] T^{\prime}(x)+\left[\alpha^{\prime}(x) J(x)+\alpha(x) J^{\prime}(x)\right] T(x)=\frac{J^{2}(x)}{\sigma(x)}+P_{a u x}(x)
\end{gathered}
$$

we get:

$m=2$

$y(x) \equiv T(x)$

- degree of the differential equation

perature

$y^{(u)}(x) \equiv T^{(u)}(x) \quad-u^{\text {th }}$ derivation of unknown temperature

$\eta_{0}(x)=\alpha^{\prime}(x) J(x)+\alpha(x) J^{\prime}(x)-0^{\text {th }}$ derivation of the temperature on the left-hand side of the differential equation

$\eta_{1}(x)=\alpha(x) J(x)-\lambda^{\prime}(x) \quad-$ non-constant coefficient of the $1^{\text {st }}$ derivation of the temperature

$\eta_{2}(x)=-\lambda(x)$

$g>0$

$\varepsilon_{j}$

- non-constant coefficient of the $2^{\text {nd }}$ derivation

- degree of the polynomial on the right-hand side

- constant coefficient of the $j^{\text {th }}$ power of the right-hand side poly-

$a_{j}(x)=\frac{x^{j}}{j !} \quad-$ auxiliary function for formulation of polynomial

Now, we can rewrite the solution of the differential equation for our case and its derivation:

$$
\begin{gathered}
T(x)=\sum_{i=0}^{1} T_{0}^{(i)} c_{i}(x)+\sum_{j=0}^{g} \varepsilon_{j} b_{j+2}(x)=c_{0}(x) T_{0}+c_{1}(x) T_{0}^{\prime}+\sum_{j=0}^{g} \varepsilon_{j} b_{j+2}(x) \\
T^{\prime}(x)=c_{0}^{\prime}(x) T_{0}+c_{1}^{\prime}(x) T_{0}^{\prime}+\sum_{j=0}^{g} \varepsilon_{j} b_{j+2}^{\prime}(x)
\end{gathered}
$$

Let us write again the constitutive equation (1) for 1D heat flux:

$$
q(x)=T(x) \alpha(x) J(x)-\lambda(x) T^{\prime}(x)
$$

Let the boundary conditions for thermal field are: 


$$
T(L)=T_{L} \quad q(0)=q_{0}
$$

Then we can put together equation (12) expressed for position $x=0$ (position at node 0 ) and equation (10) expressed for $x=L$ (node L), and using boundary conditions (13) after some mathematical operations we can write in matrix form (lower index " 0 " and " $L$ " for used physical quantities means that concerned quantity is evaluated for position $x=0$ or $x=L$, respectively):

$$
\left[c_{0}(L)+\frac{\alpha_{0} J_{0}}{\lambda_{0}} c_{1}(L)-1\right]\left[\begin{array}{l}
T_{0} \\
T_{L}
\end{array}\right]=\left[\frac{c_{1}(L)}{\lambda_{0}} q_{0}-\sum_{j=0}^{g} \varepsilon_{j} b_{j+2}(L)\right]
$$

Now, let the boundary conditions are changed:

$$
T(0)=T_{0} \quad q(L)=q_{L}
$$

Then we can put together equation (12) expressed for position $x=L$, equation (11) expressed for node L and equation (10) expressed for node L, and using boundary conditions (15) after some mathematical operations we can write in matrix form:

$$
\left[c_{0}(L)-\frac{c_{1}(L) c_{0}^{\prime}(L)}{c_{1}^{\prime}(L)} \frac{c_{1}(L) \alpha_{L} J_{L}}{c_{1}^{\prime}(L) \lambda_{L}}-1\right]\left[\begin{array}{l}
T_{0} \\
T_{L}
\end{array}\right]=\left[\frac{c_{1}(L)}{c_{1}^{\prime}(L)}\left(\frac{q_{L}}{\lambda_{L}}+\sum_{j=0}^{g} \varepsilon_{j} b_{j+2}^{\prime}(L)\right)-\sum_{j=0}^{g} \varepsilon_{j} b_{j+2}(L)\right]
$$

The matrixes (14) and (16) can be put together. Comparing mathematical formulation and FEM formulation of our task we can find out, that there is sign difference in heat flux at node L (heat flux at node L for FEM formulation has opposite direction than it is in mathematical formulation). Considering these facts we get the system of FEM equations for thermal field in the conductor:

$$
\left[\begin{array}{cc}
c_{0}(L)+\frac{\alpha_{0} J_{0}}{\lambda_{0}} c_{1}(L) & -1 \\
c_{0}(L)-\frac{c_{1}(L) c_{0}^{\prime}(L)}{c_{1}^{\prime}(L)} & \frac{c_{1}(L) \alpha_{L} J_{L}}{c_{1}^{\prime}(L) \lambda_{L}}-1
\end{array}\right]\left[\begin{array}{l}
T_{0} \\
T_{L}
\end{array}\right]=\left[\begin{array}{c}
\frac{c_{1}(L)}{\lambda_{0}} q_{0}-\sum_{j=0}^{g} \varepsilon_{j} b_{j+2}(L) \\
\frac{c_{1}(L)}{c_{1}^{\prime}(L)}\left(\frac{-q_{L}}{\lambda_{L}}+\sum_{j=0}^{g} \varepsilon_{j} b_{j+2}^{\prime}(L)\right)-\sum_{j=0}^{g} \varepsilon_{j} b_{j+2}(L)
\end{array}\right]
$$

We can also evaluate the temperature within the range of the conductor. Using equation (12) expressed for node 0 and substituting it into equation (10) then we can write:

$$
T(x)=c_{0}(x) T_{0}+c_{1}(x) \frac{T_{0} \alpha_{0} J_{0}-q_{0}}{\lambda_{0}}+\sum_{j=0}^{g} \varepsilon_{j} b_{j+2}(x)
$$

Now, comparing the general form of the differential equation (5) with governing function (4) for electric current density in expanded form:

$$
\begin{gathered}
\sum_{u=0}^{m} \eta_{u}(x) y^{(u)}(x)=\sum_{j=0}^{g} \varepsilon_{j} a_{j}(x) \\
\sigma(x) \varphi^{\prime \prime}(x)+\sigma^{\prime}(x) \varphi^{\prime}(x)=-\alpha^{\prime}(x) \sigma(x) T^{\prime}(x)-\alpha(x) \sigma^{\prime}(x) T^{\prime}(x)-\alpha(x) \sigma(x) T^{\prime \prime}(x)
\end{gathered}
$$

we get:

$m=2 \quad-$ degree of the differential equation

$y(x) \equiv \varphi(x) \quad-$ unknown function of independent variable $x$ is function of el. potential

$y^{(u)}(x) \equiv \varphi^{(u)}(x) \quad-u^{\text {th }}$ derivation of unknown electric potential

$\eta_{0}(x)=0 \quad-0^{\text {th }}$ derivation of the potential is not present on the left-hand side 


$\begin{array}{ll}\eta_{1}(x)=\sigma^{\prime}(x) & - \text { non-constant coefficient of the } 1^{\text {st }} \text { derivation of the potential } \\ \eta_{2}(x)=\sigma(x) & - \text { non-constant coefficient of the } 2^{\text {nd }} \text { derivation of the potential } \\ g>0 & \text { - degree of the polynomial on the right-hand side } \\ \varepsilon_{j} & - \text { constant coefficient of the } j^{\text {th }} \text { power of the right-hand side polynomial } \\ a_{j}(x)=\frac{x^{j}}{j !} & - \text { auxiliary function for formulation of polynomial of the right side }\end{array}$

Similar to the derivation process for thermal field we can derive also FEM equations for electric field:

$$
\left[\begin{array}{cc}
-c_{0}(L) & 1 \\
-c_{0}(L)+\frac{c_{1}(L) c_{0}^{\prime}(L)}{c_{1}^{\prime}(L)} & 1
\end{array}\right]\left[\begin{array}{l}
\varphi_{0} \\
\varphi_{L}
\end{array}\right]=\left[\begin{array}{c}
-\frac{c_{1}(L)}{\sigma_{0}} J_{0}-c_{1}(L) \alpha_{0} T_{0}^{\prime}+\sum_{j=0}^{g} \varepsilon_{j} b_{j+2}(L) \\
-\frac{c_{1}(L)}{c_{1}^{\prime}(L)}\left(\frac{-J_{L}}{\sigma_{L}}+\alpha_{L} T_{L}^{\prime}+\sum_{j=0}^{g} \varepsilon_{j} b_{j+2}^{\prime}(L)\right)+\sum_{j=0}^{g} \varepsilon_{j} b_{j+2}(L)
\end{array}\right]
$$

And similar to the equation (18) for thermal field we can also evaluate the electric potential within the range of the conductor:

$$
\varphi(x)=c_{0}(x) \varphi_{0}-c_{1}(x)\left(\frac{J_{0}}{\sigma_{0}}+\alpha_{0} T_{0}^{\prime}\right)+\sum_{j=0}^{g} \varepsilon_{j} b_{j+2}(x)
$$

The temperature and electric potential are primary variables for thermal and electric field analyses, respectively. Calculating the secondary variables, like heat flux and electric current density, is possible using equation (1) expressed for one-dimensional task. But this is suitable only for 1D model where we calculate one longitudinal distribution of the heat flux or electric current density for homogenized model, respectively. However, our 1D model results from real 3D FGM conductor, so it is more realistic to calculate the secondary variables in layers that were considered during homogenization process (see [1] for details).

FEM equations for two-way coupled thermoelectric analysis are equations (17) and (20). They are solved using iterative algorithm. During iteration process it is necessary to find substitutional functions for results obtained from FEM equations (results of these FEM equations are not continuous functions but only sets of discrete values) and also it is necessary to convert non-polynomials into polynomials (e.g. see $P_{J}$ in equation (3)). Iteration process can be set ahead by evaluating equations (1) expressed for one-dimensional task also within individual iterative steps.

\section{THERMOELECTRIC ANALYSIS OF FGM LINK CONDUCTOR - NUMERICAL EXPERIMENT}

In this chapter there will be one academic example of thermoelectric analysis of given FGM link conductor presented. The task will be solved using our new approach, by commercial FEM code ANSYS and also by numerical solution of differential equations in software Mathematica due to comparison reasons.

Let us consider electric conductor with rectangular cross-section according to Figure 1. Its length is $L=500[\mathrm{~mm}]$, height $h=10[\mathrm{~mm}]$ and width $b=20[\mathrm{~mm}]$. Let the conductor consists of mixture of two component materials - matrix (index $m$ ) with constant electric conductivity $\sigma_{m}(x, y)=1.429 \times 10^{6}\left[\mathrm{Sm}^{-1}\right]$ and thermal conductivity $\lambda_{m}(x, y)=$ $2\left[\mathrm{Wm}^{-1} \mathrm{~K}^{-1}\right]$, and fibre (index $f$ ) with electric conductivity $\sigma_{f}(x, y)=1.111 \times 10^{7}\left[\mathrm{Sm}^{-1}\right]$ and thermal conductivity $\lambda_{m}(x, y)=400\left[\mathrm{Wm}^{-1} \mathrm{~K}^{-1}\right]$. Volume fraction of individual com- 
ponents is functionally changed according to chosen polynomial, graphically shown in Figure 1:

$$
\begin{aligned}
& v_{f}(x, y)=0.7125-7.2214 x^{2}+9.6286 x^{3}+92.500 y-1658.57 x^{2} y+2211.43 x^{3} y- \\
& 2500 y^{2}+1.5514 \times 10^{5} x^{2} y^{2}-2.0686 \times 10^{5} x^{3} y^{2}-9 \times 10^{5} y^{3}+1.08 \times 10^{7} x^{2} y^{3}- \\
& 1.44 \times 10^{7} x^{3} y^{3}[-] \\
& v_{m}(x, y)=1-v_{f}(x, y)[-]
\end{aligned}
$$

Using extended mixture rule for chosen number of layers $(N=11)$ we get longitudinal variation of effective electric and thermal conductivities for individual layers and using laminate theory we can calculate also homogenized electric and thermal conductivity of FGM conductor, see Figure 4.

The equations of homogenized electric and thermal conductivities are:

$$
\begin{aligned}
& \sigma^{H}(x)=8.1273 \times 10^{6}-5.7507 \times 10^{7} x^{2}+7.6676 \times 10^{7} x^{3}\left[\mathrm{Sm}^{-1}\right] \\
& \lambda^{H}(x)=277.352-2363.82 x^{2}+3151.77 x^{3}\left[\mathrm{Wm}^{-1} \mathrm{~K}^{-1}\right]
\end{aligned}
$$

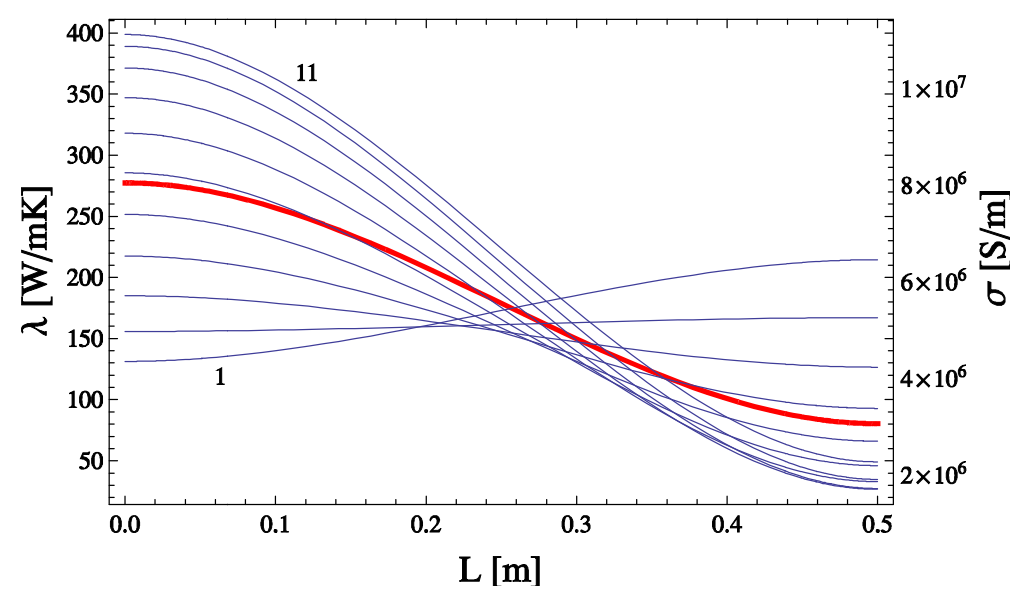

Figure 4: Longitudinal distribution of the homogenized electric and thermal conductivity (red) and the effective electric and thermal conductivities in respective layers for $N=11$ (blue)

Let us consider final Seebeck coefficient for whole conductor according to chosen polynomial function (academic example, without considering homogenization process based on mixture of the components):

$$
\alpha(x)=-4 \times 10^{-4}+28 \times 10^{-4} x^{2}\left[\mathrm{VK}^{-1}\right]
$$

We assume static state for thermoelectric analysis. In nodes 0 and $\mathrm{L}$ there are electric potentials and temperatures specified and there is variable auxiliary heat generation in the conductor, so boundary conditions (see Figure 5) are:

$$
\begin{aligned}
& \varphi(0)=0.11[\mathrm{~V}] ; T(0)=273[\mathrm{~K}] \\
& \varphi(L)=0[\mathrm{~V}] ; T(L)=283[\mathrm{~K}] \\
& P_{\text {aux }}(x)=2 \times 10^{5}-6.4 \times 10^{6} x^{5}\left[\mathrm{Wm}^{-3}\right]
\end{aligned}
$$

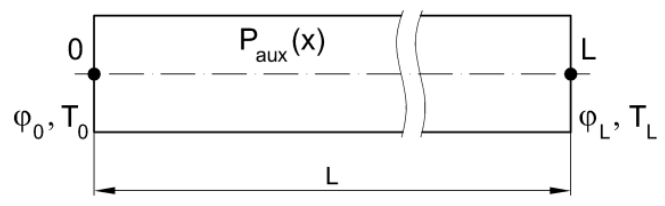

Figure 5: Boundary conditions of the model 
We also created 2D model in code ANSYS [6], we used 55000 PLANE223 elements (8 node quad-elements). The task was also solved in software Mathematica [7], where the differential equations (4) with specified boundary conditions and homogenized material properties were numerically solved using iterative algorithm. Finally, the task was also solved by only one our new developed two-nodal link element using FEM equations (17) and (20) for nodal points of the link and with equations (18) and (21) for chosen points within the link. In Figure 6 and Figure 8 we can see calculated longitudinal distribution of the electric potential and temperature in the conductor, respectively. In Figure 7 and Figure 9 there are shown distributions of the electric current densities and heat fluxes for chosen layers $\left(1^{\text {st }}, 6^{\text {th }}\right.$ and $11^{\text {th }}$ layer $)$, respectively. Summary of calculated results is in Table 1.

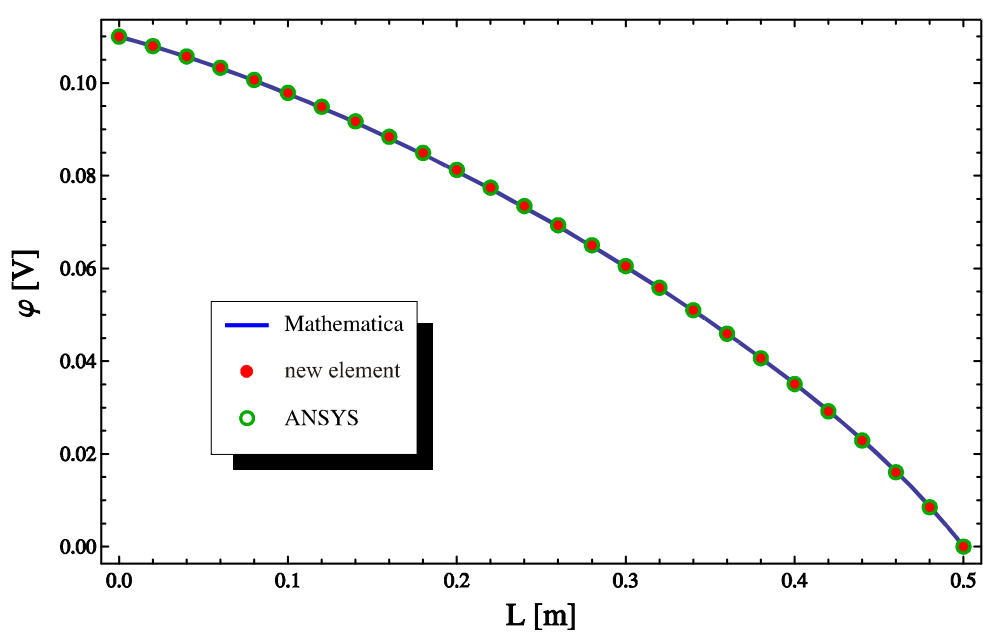

Figure 6: Distribution of the electric potential through the length of conductor

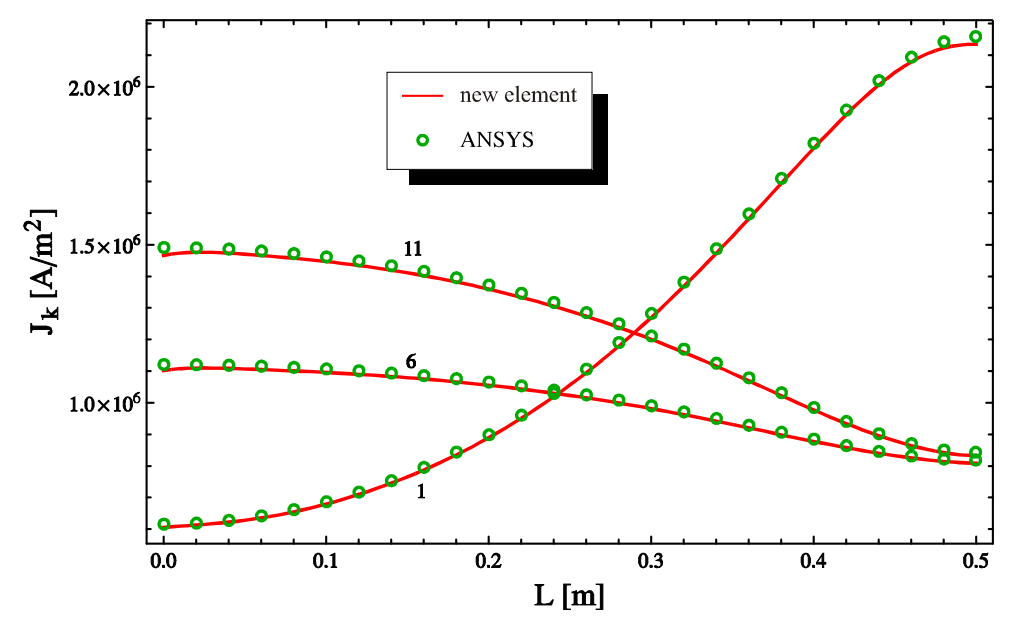

Figure 7: Longitudinal distribution of the current densities in the chosen layers of conductor 


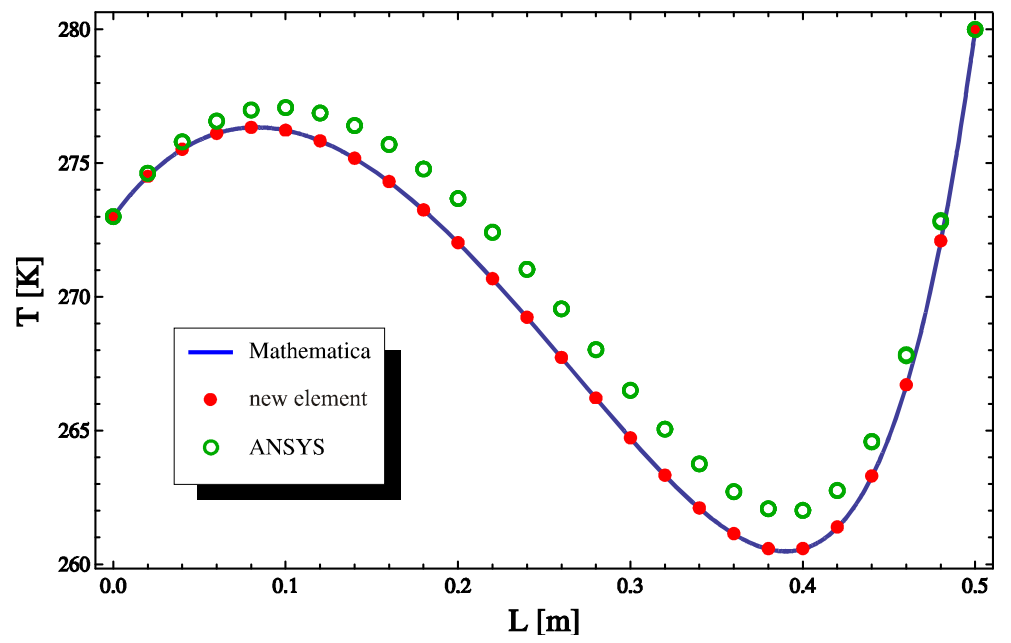

Figure 8: Distribution of the temperature through the length of conductor

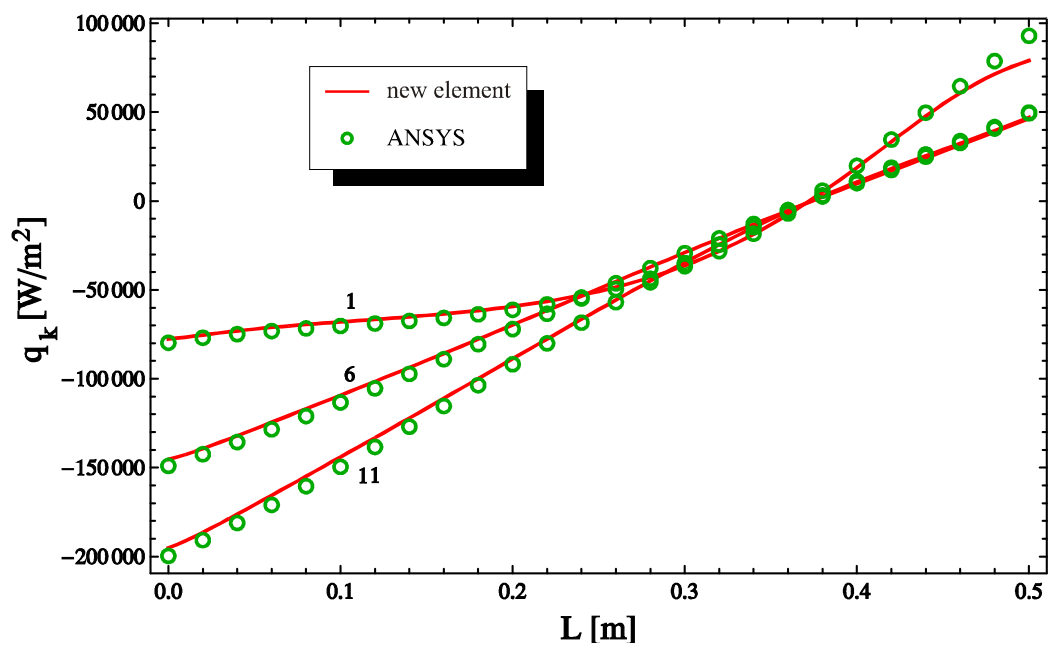

Figure 9: Longitudinal distribution of the heat fluxes in the chosen layers of conductor

\begin{tabular}{|c|c|c|c|c|c|c|c|c|}
\hline $\begin{array}{c}J_{\text {layer,node }} \\
\times 10^{5}\left[\mathrm{Am}^{-2}\right]\end{array}$ & $J_{1,0}$ & $J_{1, L}$ & $J_{6,0}$ & $J_{6, L}$ & $J_{11,0}$ & $J_{11, L}$ & $J_{0}^{H}$ & $J_{L}^{H}$ \\
\hline new element & 6.0532 & 21.3455 & 11.0189 & 8.0888 & 14.6612 & 8.3291 & 10.8288 & 10.8288 \\
\hline ANSYS & 6.1584 & 21.5903 & 11.2089 & 8.1852 & 14.9130 & 8.4328 & - & - \\
\hline Mathematica & - & - & - & - & - & - & 10.8274 & 10.8274 \\
\hline \hline $\begin{array}{c}q_{\text {layer,node }} \\
\times 10^{4}\left[W^{-2}\right]\end{array}$ & $q_{1,0}$ & $q_{1, L}$ & $q_{6,0}$ & $q_{6, L}$ & $q_{11,0}$ & $q_{11, L}$ & $q_{0}^{H}$ & $q_{L}^{H}$ \\
\hline new element & -7.7619 & 7.9057 & -14.5377 & 4.6420 & -19.5074 & 4.7011 & -14.2730 & 5.2933 \\
\hline ANSYS & -7.9756 & 9.2951 & -14.9012 & 4.9339 & -19.9653 & 4.9732 & & - \\
\hline Mathematica & - & - & - & - & - & - & -14.1887 & 5.2594 \\
\hline
\end{tabular}

Table 1: Comparison of calculated electric and thermal quantities for chosen layers and homogenized values in nodal points of the conductor

There is small difference in the results (secondary variables) between ANSYS solution and calculation using the new approach in nodal points because of substitutional functions used for conversion non-polynomials into polynomials during iterative process. But we can see 
from Figure 6 - Figure 9 that obtained results correspond to ANSYS 2D simulation very well. Differences in the results for primary variables in the conductor inner region are due to fact that our approach is based on reduction of the real 3D system into 1D problem.

\section{CONCLUSIONS}

New finite link element for two-way coupled static thermoelectric analyses has been developed in this contribution. New FEM equations with consideration Joule heat, auxiliary heat, and thermoelectric effects, like Seebeck and Peltier effects, were derived. Numerical example with good agreement between calculations with just only one new link element and commercial FEM code that uses numbers of classic elements have been presented. The new approach fully agrees with numerical solution for 1D differential equation of thermal and electric fields calculated using iterative algorithm. So, effectiveness and accuracy of the new developed link element for these analyses are excellent.

\section{ACKNOWLEDGEMENT}

This work was supported by the Slovak Research and Development Agency under the contract No. APVV-0246-12 and APVV-14-0613, by Grant Agency VEGA, grant No. 1/0228/14 and $1 / 0453 / 15$.

\section{REFERENCES}

Murín, J. et al.: Electric-Thermal Link Finite Element Made of a FGM with Spatial Variation of Material Properties. In: Composites Part B: Engineering. ISSN 1359-8368. Vol. 42 (2011), p. 1966-1979.

Rubin, H.: Analytische Lösung linearer Differentialgleichungen mit veränderlichen Koeffizienten und baustatische Anwendung. Bautechnik 76. (1999)

Murín, J., Kutiš, V.: Improved mixture rules for the composite (FGM's) sandwich beam finite element. In: Computational Plasticity IX. Fundamentals and Applications. Barcelona. (2007), p. 647-650

Altenbach, H. et al.: Mechanics of composite structural elements. Springer-Verlag. Berlin. (2003), $468 \mathrm{p}$.

Antonova, E., Looman, D.: Finite Elements for Thermoelectric Device Analysis in ASNYS. In: ICT International Conference. Clemson SC USA. (2005), p. 215-218

ANSYS Swanson Analysis System, Inc., 201 Johnson Road, Houston, PA 15342/1300, USA.

S. Wolfram MATHEMATICA 5, Wolfram research, Inc., (2003) 